\title{
Lens-induced uveitis: an update
}

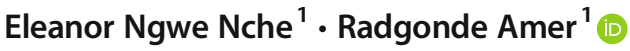

Received: 8 October 2019 / Revised: 19 December 2019 / Accepted: 30 December 2019 / Published online: 6 January 2020

(C) Springer-Verlag GmbH Germany, part of Springer Nature 2020

\begin{abstract}
Ocular inflammation resulting from a lens pathology is rare in the absence of a cataract or lens trauma because of the lens' immune privilege. The lens can be a source of ocular inflammation when the capsule is broken or when lens proteins leak out through an intact capsule. These uveitides are termed lens-induced uveitis (LIU) and are often associated with advanced cataracts. Cataracts are part of the normal aging process, and in today's world, cataract surgery is a safe and affordable means of vision restoration in the developed world. In patients with neglected cataracts and in the developing world where cataract surgery rates are lower, LIU rates are higher together with the associated complications. In this literature review, we intend to equip the armamentarium of the practicing ophthalmologist with an updated knowledge on the demographic features, clinical characteristics, treatment options, and outcomes of LIU. This is to highlight the need for timely management of cataracts before the development of advanced cataracts and LIU.
\end{abstract}

Keywords Cataract $\cdot$ Lens-induced glaucoma $\cdot$ Lens-induced uveitis $\cdot$ Phacogenic uveitis

\section{Introduction}

Patients with uveitis can present with some of the most difficult diagnostic dilemmas in all of ophthalmology. In the patient with associated lens pathology, etiologic diagnosis is even more challenging. Besides several confusing names that have been used over the years, there is a problem of cause and effect involved in the diagnostic process of these conditions. Treatment and prognosis of uveitic entities varies greatly; therefore, accurate diagnosis is imperative.

\section{Epidemiology}

Not much has been reported on the incidence and prevalence of lens-induced uveitis (LIU). Most reports on the subject are from the developing countries with low resource settings where because of low economic levels and poor access to ophthalmic centers that offer surgical treatment of cataracts,

Radgonde Amer

radgonde@gmail.com

1 Department of Ophthalmology, Hadassah Medical Center, POB 12000, 91120 Jerusalem, Israel patients present late with advanced cataracts [1]. Kothari et al. reported that $22 \%$ of patients with senile cataracts had lensinduced glaucoma (LIG) at the time of presentation to a tertiary care center in rural India [2]. Though advanced cataracts occur similarly in both sexes, LIU shows a slight female preponderance [1-3]. The reason for this is unknown.

\section{Illustrative case report}

We present the case of a 76-year-old female who presented to our clinic because of acute onset of left eye (LE) pain of 1week duration. She mentioned that she suffered from episodes of LE redness and blurry vision that had worsened over the last 3 months prior to her visit. The patient was a known hypertensive, which was controlled. She had undergone a right radical mastectomy for breast carcinoma and was currently on tamoxifen and anastrozole. In the past, she had also suffered from herpes zoster infection involving the dermatomes of the right chest.

She was pseudophakic in the right eye (RE) with Snellen's visual acuity (VA) of 0.8 and had known low vision in the LE for over 6 years due to cataract. On evaluation, RE anterior and posterior segments were unremarkable with intraocular pressure (IOP) of $9 \mathrm{mmHg}$ and a posterior chamber intraocular lens (IOL). In the LE, VA was hand movements and IOP 
was $9 \mathrm{mmHg}$. She had LE conjunctival injection, whitish precipitates on the corneal endothelium settled inferiorly, anterior chamber cells $1+$ and flare $1+$, a dense cataract with whitish precipitates on the anterior capsule, and no fundus view. B-scan ultrasonography of LE did not reveal abnormal posterior segment findings. The patient was diagnosed as a case of phacolytic uveitis and was proposed cataract surgery which she declined. She received treatment with topical prednisolone acetate $1 \%$ every hour and topical atropine $1 \%$ drops bid. Two days later, she returned with intense LE pain and an IOP of $42 \mathrm{mmHg}$. Cataract extraction by phacoemulsification was done after medically controlling IOP with posterior chamber IOL implantation. One week later, the patient felt much better; VA was 0.7 with IOP of $14 \mathrm{mmHg}$ and a quiet eye.

\section{Background}

Lens-induced uveitis (LIU) is defined as an immune reaction to lens material [4]. The pathogenesis is not fully understood but is postulated to involve breakdown of T-cell tolerance to lens protein. In 1903, Uhlenhut discovered an important characteristic of lens proteins when he injected rabbits with bovine lens proteins. Antibodies were produced and they strongly reacted with rabbit lens extracts in vitro. He concluded that lens protein was not specific to a species or individual but specific to the organ. Also, he concluded that lens protein can have the effect of an antigen in the individual where it belongs [5,6]. Verhoeff and Lemoine in 1922 coined the term endophthalmitis anaphylactica. They described 11 patients in whom extracapsular cataract extraction (ECCE) was followed by apparently sterile endophthalmitis, and most of them reacted to intracutaneous injection of lens proteins. [5-7] Following this novel description, researchers got interested in this subject, and many publications and reports followed with diverse terms used to describe and classify these conditions. The most common classification that was used in the past classified LIU into phacoanaphylactic endophthalmitis, phacotoxic uveitis, and phacolytic glaucoma [6-9]. Today, the terms phacoanaphylactic endophthalmitis and phacotoxic uveitis have been discarded since the immune reaction in LIU does not involve immunoglobulin $\mathrm{E}$ ( $\mathrm{IgE}$ ) sensitization or histamine release, neither has it been proven that lens protein is toxic to the eye. In experimental studies, a cell-mediated mechanism is postulated [6, 10-12]. LIU is now classified based on the integrity of the lens capsule. When the lens capsule is ruptured, the terms phacogenic or phacoantigenic uveitis are used, and when the lens capsule is intact, the terms phacolytic uveitis or phacolytic glaucoma are employed [13].

\section{Phacogenic uveitis}

The concept of immune privilege may apply to the lens because it is isolated from fetal circulation early in embryonic life, it lacks innervations, and the adult lens is avascular. Lens proteins can possibly be autoantigenic when present outside their position of immunological isolation in the lens capsule $[8,14]$. Each of the crystallin protein groups of lens cortical fibers contains several subtypes with distinct antigenic characteristics. The conversion process from soluble crystallins to insoluble proteins during aging of the lens can result in an increased antigenic effect of proteins of a mature or hypermature cataract $[8,15]$. The immunopathogenesis of lens-induced uveitis is believed to be the result of autosensitization to lens proteins. After a break in the lens capsule and sensitization to lens proteins, an immune complex-mediated phenomenon develops which can be transferred by hyperimmune serum $[14,16]$. This reaction can either be granulomatous or non-granulomatous and can involve a type II, type III, or type IV hypersensitivity type reaction. In a major review in 1992, Marak [17] reported that phacogenic endophthalmitis is caused by an altered tolerance of the body to lens protein and not from a rejection of sequestered lens protein. He argued that if lens proteins were treated as foreign, then phacogenic uveitis will occur more frequently after disruption of the lens capsule, but that was not the case. Many individuals have circulating lens proteins in their aqueous and do not develop an inflammatory reaction, and in rabbits, lens trauma does not always lead to ocular inflammation in aseptic conditions suggesting that the body recognizes lens proteins as self in a non-sensitized individual [16-18]. Today, LIU is recognized as a perplexing autoimmune disease resulting from an abnormal recognition of lens proteins as self [10].

Recently, the concept that tissues have immune privilege has been challenged, with studies suggesting that the brain and the eye may in fact be subject to immune surveillance and lymphatic drainage, and instead of immune privilege possess mechanisms promoting immunoquiescence [19-22].

Logan et al. showed that lens degeneration induced an immune response resulting in immune cells populating the lens that contributed to the development of fibrosis. [23] This was demonstrated in an experimental mouse model using an $\mathrm{N}$ cadherin lens-specific conditional knockout mouse, $\mathrm{N}$ $\mathrm{cad}^{\Delta \text { lens }}$ in which loss of this cell-cell junctional protein caused lens degeneration, necrosis, and fibrotic change, postnatally. Also, the authors demonstrated that the lens was connected to the lymphatic system along the suspensory ligaments that connect the lens to the ciliary body, providing a potential mechanism for the immune circulation.

Another important finding was that lens degeneration activated an immune response throughout the eye, including cornea, vitreous humor, and retina, suggesting a coordinated 
protective response in the visual system to defects of a component tissue. These studies unveiled that, contrary to prior beliefs, the lens is subject to immune surveillance [23].

Very little is known about the prevalence of phacogenic uveitis. Chung et al. reported a prevalence of $0.4 \%$ in their study in a Korean population [24]. Phacogenic uveitis tends to occur in the elderly with a peak at $60-70$ years of age; however, traumatic cataracts have been described more in the young $[17,25]$. The average time of onset of symptoms is within 2 weeks of lens trauma or intraocular surgery with a range from 2 days to 59 years, and the course can be mild and indolent or severe and fulminant $[25,26]$. Kang et al. described a unique case of an anterior uveitis in a patient with a retained lens fragment presenting 15 years after phacoemulsification [27]. Patients present with a sudden or insidious onset of ocular pain, decreased vision, redness, and photophobia. Signs on clinical evaluation correlate with an anterior uveitis that can be granulomatous or non-granulomatous [17]. In the early 1990s, only $5 \%$ of histologically confirmed cases were diagnosed clinically posing a dilemma for the ophthalmic clinician $[17,26]$. Visual acuity is variable as well as IOP. Patients can have normal IOP, ocular hypertension, or hypotension. Findings on slit-lamp examination include conjunctival congestion, corneal edema, keratic precipitates (KPs) of varying sizes mostly of the "mutton-fat" type, posterior synechiae, hypopyon, retained lens fragment in the anterior chamber, debris or lens fragments in the anterior chamber angle, pupillary membrane, vitritis, and even a panophthalmitis [7, 26, 28]. Rathinam et al. first reported the occurrence of spontaneous hyphema and acute ocular hypertension in patients with severe LIU in the absence of trauma or ocular neovascularization. [29] The authors speculated that the severe inflammation in LIU resulted in severe vascular compromise that led to extravasation of both white and red blood cells causing hypopyon and spontaneous hyphema formation.

Though classically an anterior uveitis, up to $76 \%$ of patients have an associated nonspecific choroiditis [26]. Histopathology demonstrates a zonal granulomatous inflammation centered around the site of lens capsule rupture with the presence of neutrophils and the lens material surrounded by lymphocytes, plasma cells, histiocytes, epithelioid cells, and macrophages $[17,25,26]$.

\section{Phacolytic glaucoma}

Flocks et al. in 1955 coined the term phacolytic glaucoma, describing glaucoma resulting from leakage of lens material into the anterior chamber from hypermature cataracts [28]. In 1967 Goldberg demonstrated the presence of large rounded macrophages in the aqueous humor of these patients, and
2 years later, Duke-Elder reported the presence of phagocytes around the lens capsule and macrophages stuck in trabecular meshwork [30]. As the lens ages, lens proteins become altered and denatured resulting in an increase in high molecular weight proteins. These proteins are soluble and leak through an intact permeable lens capsule into the anterior chamber where an inflammatory reaction takes place. Lens proteins, cells, and debris can move into the anterior chamber angle where they subsequently block the trabecular meshwork and cause an increase in IOP and hence a secondary glaucoma with an open angle. Phacolytic glaucoma has been described more in the elderly population due to the high prevalence of hypermature cataracts in this age group. The typical patient has a history of poor vision from the long-standing cataract and presents with an acute onset of ocular pain, redness, and worsening vision [7, 31]. Ocular tension in these patients can range from normal to values as high as $90 \mathrm{mmHg}$. Clinical signs include conjunctival hyperemia, corneal edema, prominent anterior chamber cells and flare, an open anterior chamber angle, a pseudohypopyon, and a hypermature or morgagnian cataract. The presence of protein clumps and cell debris is often confused for KPs, and the examiner should pay special examination on slit-lamp examination and gonioscopy to differentiate between them. Crystals in the anterior and posterior chamber have been described as well as spontaneous hyphema with no other associated causes of rubeosis iridis and no previous history of trauma $[29,32]$.

\section{Diagnosis}

LIU is often a clinical diagnosis though challenging. Only six of a hundred and forty cases were diagnosed clinically in one review [26]. Confirmatory assessments include needle biopsy and histopathology to detect a zonal granulomatous reaction surrounding disorganized lens material. Tanito et al. used western blot for lens- specific protein in the aqueous to confirm the diagnosis of post-traumatic LIU. The authors found higher titers of alpha and beta crystallin in their patient compared to a control group of cataract patients without lens trauma. Aqueous humor cytology is also helpful in elucidating the correct diagnosis in patients with an anterior uveitis of unknown etiology [33, 34]. Clinicians should assist the patient in recalling a possible remote incident of ocular trauma which may have been forgotten. Determining the status of the lens capsule whether integral or disrupted is crucial in the diagnostic process. Where available, scanning electron microscopy can be used to detect disruptions of the anterior lens capsule [35]. Optical coherence tomography (OCT) can be used to detect the integrity of the lens capsule and subcapsular fluid pockets in morgagnian cataracts $[36,37]$. 


\section{Differential diagnosis of LIU}

Given that several forms of uveitis can present with variable severity including an anterior chamber reaction with or without hypopyon, one should consider other causes of uveitis given the circumstances of onset and presentation of every individual patient. The wide spectrum of differential diagnoses includes post-traumatic endophthalmitis, postoperative endophthalmitis, sterile endophthalmitis, traumatic iritis, herpetic uveitis, anterior uveitis, intermediate uveitis, and PosnerSchlossman syndrome [7, 17]. Sympathetic ophthalmia can be confused with LIU, but in the latter, the fellow eye always remains quiet, a situation not seen in sympathetic ophthalmia [7].

\section{Treatment}

There has been an evolution in the management of LIU over the years; however, removal of the inciting lens material is the mainstay of treatment of all forms of LIU [5, 17, 38]. Treatment is tailored to the clinical presentation of every case. Cataract surgery, anterior chamber washout, or pars plana vitrectomy are used to extract the lens or retained lens particles $[7,17,39]$. Conservative management of lens particles in the vitreous with topical steroids is recommended if these particles are small and resolving without destructive inflammation [17]. Early lens removal leads to better visual outcomes [1, 25].

\section{Enucleation}

In some reports, enucleation was described as a method of managing LIU especially when the visual prognosis was low or absent [7, 25, 40, 41]. Johnson et al. reported a case of a male in his 60 s who presented with LIU in addition to harboring a large intraocular inflammatory mass with features of both myofibroblastic tumor and fibrous histiocytoma [41]. Enucleation was performed as the eye was blind and painful with vision of no light perception and led to an excessive removal of eyes with visual potential. In a pathologic study of eyes enucleated because of glaucoma associated to a hypermature cataract, Flocks et al. reported that 21 out of 138 eyes enucleated had a correct clinical diagnosis of LIG, and none of them had been offered cataract extraction which could have been vision-saving. [28]

\section{Lens extraction}

Verhoeff's aphorism was that it is better to remove the offending lens or residual lens material than to remove the eye [7]. LIU with the associated thinning of the lens capsule, the weak zonules and elevated IOP, makes cataract extraction challenging. Initially, intracapsular cataract extraction (ICCE) with copious anterior chamber irrigation was the mainstay of treatment as it was believed that a complete removal of the lens including the capsule was necessary for inflammation control $[28,42]$. With the advancement in cataract surgery techniques, there was a gradual move to extracapsular cataract extraction (ECCE) with some authors advocating for posterior chamber IOL insertion in the same surgery. Although IOP was raised in some cases in the early postoperative period, all cases resulted in improvement in visual acuity (at least 20/50), normalized IOP, and inflammation control [43-46]. Phacoemulsification is technically difficult to perform in patients with LIU as the thin and friable capsule in these patients makes a continuous curvilinear capsulorhexis difficult to perform, and the mature cataracts are hard to emulsify thus increasing the risk for complications like posterior capsule rupture, zonule dialysis, and endothelial cell damage [47]. Manual small-incision cataract surgery (MSICS) is a safe and efficient treatment method for LIU. Its postoperative complications are comparable to that of ECCE/IOL and phacoemulsification [48]. Compared to ECCE, MSICS offers better visual results. Venkatesh et al. reported that $78.8 \%$ of their patients had a BCVA of 20/40 or better by the 40th postoperative day [49]. MSICS results in less astigmatism and risk for expulsive hemorrhage with a smaller incision compared to ECCE and offers less cost and an easier technique in experienced hands compared to phacoemulsification $[48,49]$. In developing countries where the burden of advanced cataracts and associated complications like LIU lies, MSICS is a cost-effective option for the management of LIU.

\section{Intraocular pressure control}

Adjuvant treatments include mydriatics and IOP lowering agents. Elevated IOP in LIU is not only a risk for more surgical complications, but sometimes persists after surgery and because of damage to the optic nerve affects postoperative visual outcomes. Before surgery, osmotics, carbonic anhydrase inhibitors, and timolol eye drops can be used to control IOP [3, 50].

Combined trabeculectomy and cataract surgery leads to significant reduction in IOP and reduces the need for systemic hypotensives. When compared to cataract surgery alone, VA and IOP were similar at 6 months postoperatively in both groups [51]. Peripheral iridotomy, iridectomy, and cyclodiathermy have also been used to relieve ocular hypertension [7].

\section{Anti-inflammatory treatment}

\section{Steroids}

Corticosteroids are potent inhibitors of the products of the cyclooxygenase and lipo-oxygenase pathways. Their control 
of LIU is achieved through their anti-inflammatory effects or through the inhibition of antibody synthesis [4, 40]. In experimental studies, systemic methylprednisolone, hydrocortisone, and prednisone have been used successfully to control the inflammation in LIU $[7,52,53]$. Topical corticosteroids reduce intraocular inflammation by inhibiting the infiltration of monocytes and macrophages that are recruited by the chemotactic effects of leaking lens proteins. Also, they act at inhibiting the formation of pro-inflammatory cytokines from arachidonic acid [7, 30]. Though small retained lens particles can be managed with steroids only, they are often used to quieten the eye pending lens extraction [7, 25].

\section{Others}

Intravenous typhoid therapy has been historically used to control inflammation in LIU eyes [7]. Allopurinol controlled the ocular inflammation in LIU in mice [52-54]. AlphaChymotrypsin was used in association to sectoral iridectomy to control the intense inflammation that sometimes resulted after lens extraction. [50]

\section{Associations}

There are several reports of LIU occurring with other ocular conditions contributing to the degree of ocular inflammation seen in specific cases.

Wang et al. reported a rare case of LIU in a patient with choroidal melanoma. The authors hypothesized that the prolonged intraocular inflammation induced by the tumor led to the formation of a pupillary membrane and subsequent disruption of the lens capsule, resulting in leakage of lens protein and sterile panuveitis [55]. Johnson et al. described an inflammatory tumor with characteristics of both myofibroblastic tumor and fibrous histiocytoma [41]. In $3 \%$ to $23 \%$ of cases of LIU, there is an associated sympathetic ophthalmia [7, 17, 38]. A case of metastatic bacterial endophthalmitis after infectious pneumonia occurred with LIU. The authors theorized that chronic ocular inflammation can lead to a contracture and breakage of the lens capsule and subsequent LIU. This mechanism has also been described in patients with Behçet's disease [56]. In a review of 29 cases with LIU in patients less than 55 years of age and no history of trauma, $29 \%$ were reported to have microphthalmia in the involved eye. The authors theorized about the existence of an associated persistent hyperplastic primary vitreous (PHPV) that resulted in early capsule rupture and cataract. LIU has also been reported in patients with lens dislocation into the vitreous with no compromise of the lens capsule [7].

Phacolytic uveitis in children can be associated with congenital cataracts. Vandienbroucke et al. reported the case of a 9-day-old baby with bilateral congenital cataracts and severe granulomatous anterior uveitis resolving only after bilateral lens extraction. Extensive infectious and metabolic workup was negative [39].

\section{Outcomes}

Timely intervention leads to good visual recovery as delays in intervention lead to further ocular damage and risks for complications during surgery $[7,25]$. Patients who have longer delays between the onset of symptoms and time of cataract extraction (usually more than 2 weeks) have poorer visual outcomes and higher rates of surgical complications [2]. Up to $60 \%$ of patients with late presentation retain a vision of $<6$ / 60 after cataract surgery. [2] This highlights the need for timely cataract surgery and prompt cataract extraction when the diagnosis of a LIU is made. Reasons for poor visual outcomes on late presentation are associated optic disc atrophy, anterior uveitis, and corneal edema [1].

Regarding the type of method used for cataract extraction, phacoemulsification is known to be technically more difficult in advanced and mature cataracts with associated higher complication rates except in the hands of experienced surgeons [46]. There are currently no reports on the visual outcomes of phacoemulsification in LIU. Several authors report on the use of ECCE and MSICS for cataract extraction in phacolytic glaucoma [3, 47, 49]. MSICS however offers better visual results and less complication rates with up to $78.8 \%$ of patients achieving a postoperative best-corrected visual acuity of at least $6 / 12$ or better [47].

\section{Conclusion}

LIU is a group of entities that cause intraocular inflammation resulting from an inciting lens. Over the years several confusing terms have been used in its description and today a simplified nomenclature system based on the integrity of the lens capsule is used to define etiology, which can either be phacogenic or phacolytic. The aging cataractous lens is implicated in the development of LIU; thus, prevalence is higher in populations with an unmet need for cataract surgery like in the developing world. Besides temporizing measures like IOP reduction, and anti-inflammatory treatment, lens extraction is the definitive means of uveitis control in these patients. MSICS with IOL insertion is a safe and efficient method in the management of LIU offering better visual outcomes compared to ICCE and ECCE that were used historically. Although phacoemulsification and IOL insertion is the standard of care in the management of cataracts today, there are no reports on its use and outcomes in the management of LIU. This review aims at raising awareness on the devastating complications linked to neglected cataracts, and the need for 
prompt and adequate management of LIU before the onset of visual compromises due to longstanding ocular inflammation.

\section{Method of literature search}

The authors conducted a search of Medline (from 1960 to 2016) and ISI (from 1986 to 2016) databases using the following keywords: cataract, lens-induced glaucoma, lensinduced uveitis, phacogenic uveitis, phacoanaphylactic endophthalmitis, and phacotoxic uveitis. In addition, references quoted in the articles found through these databases were also included, if found appropriate. All articles judged to be of clinical relevance to the topic were included. For articles in languages other than English, abstracts were evaluated and, when appropriate, also included.

\section{Compliance with ethical standards}

Conflict of interest The authors declare that they have no conflict of interest.

Ethical approval This article does not contain any studies with human participants or animals performed by any of the authors.

\section{References}

1. Sitoula RP, Sarkar I, Nayak D, Singh SK (2016) Lens induced glaucoma: an experience in tertiary eye care center in eastern Nepal. Nepal J Ophthalmol 8(16):161-166

2. Kothari R, Tathe S, Gogri P, Bhandari A (2013) Lens-induced glaucoma: the need to spread awareness about early management of cataract among rural population. ISRN Ophthalmol 2013:581727

3. Sharanabasamma M, Vaibhav K (2016) Management and visual outcome in patients of lens-induced glaucomas at a tertiary eye care hospital in South India. J Curr Glaucoma Pract 10(2):68-75

4. Wirostko E, Spalter HF (1967) Lens-induced uveitis. Arch Ophthalmol 78(1):1-7

5. Riise P (1965) Endophthalmitis phacoanaphylactica. Am J Ophthalmol 60(5):911-915

6. Irvine SR, Irvine AR Jr (1952) Lens-induced uveitis and glaucoma. I Endophthalmitis phaco-anaphylactica Am J Ophthalmol 35(2): 177-186

7. Chandler PA (1958) Problems in the diagnosis and treatment of lens-induced uveitis and glaucoma. AMA Arch Ophthalmol. 60(5):828-841

8. Irvine SR, Irvine AR Jr (1952) Lens-induced uveitis and glaucoma. III. "Phacogenetic glaucoma": lens-induced glaucoma; mature or hypermature cataract; open iridocorneal angle. Am J Ophthalmol 35(4):489-499

9. Hammer H, Olah M (1974) Cellular hypersensitivity to lenticular protein in lens-induced uveitis. Albrecht Von Graefes Arch Klin Exp Ophthalmol 192(4):339-344

10. Margo CE, Harman LE (2016) Autoimmune disease: conceptual history and contributions of ocular immunology. Surv Ophthalmol 61(5):680-688

11. Irvine SR, Irvine AR Jr (1952) Lens-induced uveitis and glaucoma. Part II. The "phacotoxic" reaction. Am J Ophthalmol 35:370
12. Lai JC, Lobanoff MC, Fukushima A, Wawrousek EF, Chan CC, Whitcup SM, Gery I (1999) Uveitis induced by lymphocytes sensitized against a transgenically expressed lens protein. Invest Ophthalmol Vis Sci 40(11):2735-2739

13. Basic and Clinical Science Course. Section 9 (2016-2017) Intraocular inflammation and uveitis. American Academy of Ophthalmology Pages 118-119

14. Halbert SP (1965) Manski W. Biological aspects of autoimmune reactions in the lens Invest Ophthal 4:516-530

15. Sanber HO (1976) The alpha-crystallin content of aqueous humour in cortical, nuclear, and complicated cataracts. Exp Eye Res 22(1): 75-84

16. Marak GE Jr, Font RL, Czawlytko LN, Alepa FP (1974) Experimental lens-induced granulomatous endophthalmitis: preliminary histopathologic observations. Exp Eye Res 19(4):311316

17. Marak GE Jr (1992) Phacoanaphylactic endophthalmitis. Surv Ophthalmol 36(5):325-339

18. Farman C, Schuetz C, Lorget F, Bantseev V, Calise D, Thackaberry EA (2017) Lens. Capsule Perforation Without Inflammation in 4 Rabbits From Intravitreal Injection Studies Vet Pathol 54(5):870 876

19. Aspelund A, Antila S, Proulx ST, Karlsen TV, Karaman S, Detmar M, Wiig H, Alitalo K (2015)A dural lymphatic vascular system that drains brain interstitial fluid and macromolecules. J Exp Med. 212: 991-9

20. Knickelbein JE, Buela KA, Hendricks RL (2014) Antigenpresenting cells are stratified within normal human corneas and are rapidly mobilized during ex vivo viral infection. Invest Ophthalmol Vis Sci 55:1118-1123

21. Ransohoff RM, Engelhardt B (2012) The anatomical and cellular basis of immune surveillance in the central nervous system. Nat Rev Immunol 12:623-635

22. Shechter R, London A, Schwartz M (2013) Orchestrated leukocyte recruitment to immune-privileged sites: absolute barriers versus educational gates. Nat Rev Immunol. 13:206-218

23. Logan CM, Bowen CJ, Menko AS (2017) Induction of immune surveillance of the dysmorphogenic lens. Sci Rep 7:16235

24. Chung H, Choi DG (1989) Clinical analysis of uveitis. Kor J ophthalmol 3:33-37

25. Perlman EM, Albert DM (1977) Clinically unsuspected phacoanaphylaxis after ocular trauma. Arch Ophthalmol 95(2): 244-246

26. Thach AB, Marak GE Jr, McLean IW, Green WR (1991) Phacoanaphylactic endophthalmitis: a clinicopathologic review. Int Ophthalmol 15(4):271-279

27. Kang HM, Park JW, Chung EJ (2011) A retained lens fragment induced anterior uveitis and corneal edema 15 years after cataract surgery. Korean J Ophthalmol 25(1):60-62

28. Flocks M, Littwin CS, Zimmerman LE (1955) Phacolytic glaucoma; a clinicopathologic study of one hundred thirty-eight cases of glaucoma associated with hypermature cataract. AMA Arch Ophthalmol 54(1):37-45

29. Rathinam SR, Cunningham ET Jr (2010) Spontaneous hyphaema and acute ocular hypertension associated with severe lens-induced uveitis. Eye (Lond) 24(12):1822-1824

30. Sood GC, Sofat BK, Chandel RD, Mehrotra SK (1972) Prognosis in spontaneous phakolytic glaucoma. Br J Ophthalmol 56(8):621623

31. Rosenbaum JT, Samples JR, Seymour B, Langlois L, David L (1987) Chemotactic activity of lens proteins and the pathogenesis of phacolytic glaucoma. Arch Ophthalmol 105(11):1582-1584

32. Peracha-Riyaz MH, Peracha ZH, Spaulding J, Baciu P, Ahmed S, Imami NR, Darnley-Fisch D, Desai U (2017) First described case of anterior and posterior segment crystals in phacolytic glaucoma. $\mathrm{J}$ Glaucoma 26(5):e171-e173 
33. Tanito M, Kaidzu S, Katsube T, Nonoyama S, Takai Y, Ohira A (2009) Diagnostic western blot for lens-specific proteins in aqueous fluid after traumatic lens-induced uveitis. Jpn J Ophthalmol 53(4): 436-439

34. Kalogeropoulos CD, Malamou-Mitsi VD, Asproudis I, Psilas K (2004) The contribution of aqueous humor cytology in the differential diagnosis of anterior uvea inflammations. Ocul Immunol Inflamm 12(3):215-225

35. ten Doesschate MJ (1985) Lens-induced inflammation. Int Ophthalmol 7(3-4):193-201

36. Dhami A, Dhami AS, Singh H, Dhami GS (2019) Role of anterior segment optical coherence tomography for safer management of mature white cataracts. J Cataract Refract Surg 45(4):480-484

37. Tabatabaei A, Hasanlou N, Kheirkhah A, Mansouri M, Faghihi H, Jafari H, Arefzadeh A, Moghimi S (2014) Accuracy of 3 imaging modalities for evaluation of the posterior lens capsule in traumatic cataract. J Cataract Refract Surg 40(7):1092-1096

38. Yoo WS, Kim BJ, Chung IY, Seo SW, Yoo JM, Kim SJ (2014) A case of phacolytic glaucoma with anterior lens capsule disruption identified by scanning electron microscopy. BMC Ophthalmol 14: 133

39. Vandenbroucke S, Foets B, Wouters C, Casteels I (2014) Bilateral congenital cataract with suspected lens-induced granulomatous uveitis. J AAPOS 18(5):492-494

40. Luntz MH, Wright R (1962) Lens-induced uveitis. Exp Eye Res 1: $317-323$

41. Johnson JG, Margo CE (2017) Intraocular inflammatory mass associated with lens-induced uveitis. Surv Ophthalmol 62(4):541545

42. Ellant JP, Obstbaum SA (1992) Lens-induced glaucoma. Doc Ophthalmol 81(3):317-338

43. Lane SS, Kopietz LA, Lindquist TD, Leavenworth N (1988) Treatment of phacolytic glaucoma with extracapsular cataract extraction. Ophthalmology 95(6):749-753

44. Singh G, Kaur J, Mall S (1994) Phacolytic glaucoma-its treatment by planned extracapsular cataract extraction with posterior chamber intraocular lens implantation. Indian J Ophthalmol 42(3):145-147

45. McKibbin M, Gupta A, Atkins AD (1996) Cataract extraction and intraocular lens implantation in eyes with phacomorphic or phacolytic glaucoma. J Cataract Refract Surg 22(5):633-636
46. Mandal AK (1996) Endocapsular surgery and capsular bag fixation of intraocular lenses in phacolytic glaucoma. J Cataract Refract Surg 22(3):288-293

47. Vajpayee RB, Angra SK, Honavar SG, Katoch S, Prasad N, Bansal A, Anand J (1995) Capsulotomy for phacoemulsification in hypermature cataracts. J Cataract Refract Surg 21(6):612-615

48. Venkatesh R, Chang DF, Muralikrishnan R, Hemal K, Gogate P, Sengupta $S$ (2012) Manual small incision cataract surgery: a review. Asia Pac J Ophthalmol (Phila) 1(2):113-119

49. Venkatesh R, Tan CS, Kumar TT, Ravindran RD (2007) Safety and efficacy of manual small incision cataract surgery for phacolytic glaucoma. Br J Ophthalmol 91(3):279-281

50. Epstein DL (1982) Diagnosis and management of lens-induced glaucoma. Ophthalmology 89(3):227-230

51. Braganza A, Thomas R, George T, Mermoud A (1998) Management of phacolytic glaucoma: experience of 135 cases. Indian J Ophthalmol 46(3):139-143

52. Augustin AJ, Spitznas M, Sekundo W, Koch F, Lutz J, Meller D, Grus FH, Wegener A, Blumenröder SH (1996) Effects of allopurinol and steroids on inflammation and oxidative tissue damage in experimental lens induced uveitis: a biochemical and morphological study. Br J Ophthalmol 80(5):451-457

53. Grus FH, Augustin AJ, Zimmermann CW (1997) Computersupported analysis (MegaBlot) of allopurinol-induced changes in the autoantibody repertoires of rats suffering from experimental lens-induced uveitis. Electrophoresis 18(3-4):516-519

54. Augustin AJ, Böker T, Blumenröder SH, Lutz J, Spitznas M (1994) Free radical scavenging and antioxidant activity of allopurinol and oxypurinol in experimental lens-induced uveitis. Invest Ophthalmol Vis Sci 35(11):3897-3904

55. Wang LC, Kim JW, Rao NA (2018) Coexisting phacoanaphylaxis and choroidal melanoma: an unusual intraocular inflammation. Ocul Immunol Inflamm 26(8):1278-1282

56. Murase KH, Goto H, Kezuka T, Yamauchi Y, Usui M (2007) A case of lens-induced uveitis following metastatic endophthalmitis. Jpn J Ophthalmol 51(4):304-306

Publisher's note Springer Nature remains neutral with regard to jurisdictional claims in published maps and institutional affiliations. 